\title{
Towards Using Sensors as Data Sources in Teaching: Requirements for School Curricula-Compatible Sensors
}

\author{
Mubashrah Saddiqa $^{1 *}$, Marite Kirikova ${ }^{2}$, Rikke Magnussen ${ }^{3}$, \\ Birger Larsen ${ }^{3}$, and Jens Myrup Pedersen ${ }^{1}$ \\ ${ }^{1}$ Department of Electronic Systems, Aalborg University, Aalborg, Denmark \\ ${ }^{2}$ Institute of Applied Computer Systems, Riga Technical University, Riga, Latvia \\ ${ }^{3}$ Department of Communication and Psychology, Aalborg University, Copenhagen, Denmark \\ mus@es.aau.dk, marite.kirikova@rtu.lv, rikkem@hum.aau.dk, \\ birger@hum.aau.dk, jens@es.aau.dk
}

\begin{abstract}
The continuous innovation in modern technologies in various sectors of society has transformed everyday life. It becomes imperative for the educational community to equip the future generation with digital skills. In this article, using qualitative and quantitative research techniques, we define criteria for school curricula-compatible sensors (particularly, for secondary school students in grades 5 through 9). We also develop requirements models for sensor classes that satisfy the school curricula compatibility criteria using requirements engineering techniques. The results show that integrating sensors into schools can improve students' digital and data skills. Additionally, requirements models can help in developing school curricula-compatible sensors or transforming existing sensors into curricula-compatible sensors.

Keywords: School Education, Sensors, Digital Skills, Data Skills Requirements for Physical Systems, Technology Requirements.
\end{abstract}

\section{Introduction}

The importance of sensor technology is growing continuously. Using sensor technology, we can explore and monitor our surroundings in ways that were not possible even a few years ago. New sensor technology variations and applications are advancing rapidly and expanding their scope and impact on everyday life. Sensors that are simple to use and handle can be used in a variety of domains, including education, rather than just technical domains. Among the advantages of sensor technology's use in education is that it provides students with a means of observing, interpreting, and investigating different phenomena in real time [1]. The hands-on methods

\footnotetext{
* Corresponding author
}

(C) 2021 Mubashrah Saddiqa, Marite Kirikova, Rikke Magnussen, Birger Larsen, and Jens Myrup Pedersen. This is an open access article licensed under the Creative Commons Attribution License (http://creativecommons.org/licenses/by/4.0).

Reference: M. Saddiqa, M. Kirikova, R. Magnussen, B. Larsen, and J. M. Pedersen, "Towards Using Sensors as Data Sources in Teaching: Requirements for School Curricula-Compatible Sensors," Complex Systems Informatics and Modeling Quarterly, CSIMQ, no. 26, pp. 78-93, 2021. Available: https://doi.org/10.7250/csimq.2021-26.05

Additional information. Author ORCID iD: M. Saddiqa - https://orcid.org/0000-0002-4816-2426, M. Kirikova https://orcid.org/0000-0002-1678-9523, R. Magnussen - https://orcid.org/0000-0001-8058-9027, B. Larsen https://orcid.org/0000-0002-3622-2698, and J. M. Pedersen - https://orcid.org/0000-0002-1903-2921. PII S225599222100152X. Received: 15 March 2021. Accepted: 26 April 2021. Available online: 30 April 2021. 
involved in using sensor technology to collect and analyze data also seem useful for students who find math and science lessons difficult [2]. Data-collection activities and experiments encourage critical thinking, allowing students to engage in real investigations rather than prescriptive experiments that have pre-determined outcomes.

Bringing sensors into schools will not only help students in addressing the real problems of their own neighborhoods and surroundings but also attract students' attention and motivate involvement in school lessons. Using sensors in the teaching can motivate students to follow science subjects. Their use also provides students with a means to determine how they can collect data about real world surrounding them and how they can make decisions based on the collected data, e.g., about the classroom environment, traffic flows outside their schools, pollution levels, etc.

An alternative to sensor data is open data, which is becoming increasingly available. In school subjects, open data can also provide real information about students' surroundings and cities using simple visualizations and graphs [3]. However, the open data concept itself can be obscure to students, and they need hands-on experience of why and how data is generated and how the data can be analyzed and interpreted. With data-collection activities using sensors, students can collect data and analyze it as part of their lessons. Students can also collect sensor data on relevant topics, e.g., pollution levels around the school area, and compare it with available open data, for instance, data provided via the Open Data Interface ${ }^{2}$ designed for Danish public schools [4].

The integration of sensor technology into the curricula is not only propitious to several ways of instruction and learning but can also be beneficial in understanding and investigating the immediate surroundings of the students [5]. A fundamental obstacle in learning data science, especially at the school level, is that children do not understand how science is relevant to them [6]. Sensor technology can play an important role to help children understand more about their physical and material environment. However, for the successful use of sensors in schools as part of everyday teaching, we need to define criteria for the design of curricula-compatible sensors. We will investigate the following research question: "How can sensors facilitate the understanding of data and working with real world data, and how can sensors be designed for use in education?".

This article makes the following main research contributions:

1. It identifies several possibilities in which the use of sensors can help students learn digital and data skills.

2. It identifies main classes of school curricula-compatible sensors.

3. It proposes requirements models that help identifying attributes relevant for school curricula-compatible sensors.

Our study is part of the Community Drive project, ${ }^{3}$ which is aimed at secondary school students aged 11-15 years, with a particular focus on data and technology integration into schools. We will thus use the term students for secondary school students aged 11-15 in the rest of the article. The article is organized as follows. In Section 2, the background of sensors' use in schools is discussed. Section 3 presents the research design for defining the criteria and developing requirements for curricula-compatible sensor classes. Section 4 presents the results of our one-on-one interviews, focus group study, and pilot tests with key stakeholders, i.e., teachers and students. Finally, Section 5 presents a short discussion and the conclusions.

\section{Background}

In this digital era, the younger generation of school students needs real-world skills to address the needs they will face in the future, such as critical thinking, collaboration, discussion, creativity, teamwork, imagination, and problem-solving [7]. Sensor technology can change the

\footnotetext{
${ }^{2}$ https://odw.aau.dk/

${ }^{3}$ https://www.communitydrive.aau.dk/
} 
traditional ways of teaching science in schools by changing the learning environment and advancing the teaching methods of science and math [8]. A sensor is generally defined as "a device that detects or measures a physical property and records, indicates or responds to it" [9]. Sensors are designed to generate data, and the data is used to examine and find solutions to problems in our surroundings. Using sensor technology, students can become more creative and develop their social, linguistic, cognitive, visual, and emotional skills [10], [11], [12].

Researchers have discussed various studies of integrating sensors and their advantages in educational activities within secondary and primary schools. For instance, [13] reports positive experiences of using sensors in a primary schools with students aged 7-8 years in the UK. The authors used probes and data-logging software to introduce dynamic line graphs to young children from the science investigations perspective. Additionally, [14] discusses a case study in which first grade students collected weather data during the school year and designed several graphs to represent their data. The results show a high level of interest in data-collection experiments and describe how first grade students worked with the graphs and maps. Other positive experiences have been reported in well-controlled studies by [15] in Cyprus and by [16] in the USA. The results show how children can handle data logging equipment and learn with it.

From another perspective, many researchers, e.g., [17], [18] have shown that students lose interest and self-confidence in math and other science subjects. However, by using sensors and technology, these subjects could become interesting in primary and K-12 school systems. A paper by Grufberg and Jonsson, 2012, [19] discusses a way to motivate children to learn about science by creating a design process scenario. The resulting design concepts demonstrated several innovative uses of sensor technology, indicating students' understanding of the technology and the natural phenomena under consideration. The sensor oriented activities also encouraged discussions and reflections around the abstract concepts.

A formal study [20] conducted in Cape Town, South Africa used a commercially available technology toy, littleBits. The authors explored the potential of littleBits as a learning tool for computational thinking in three different educational settings. The findings show that, in addition to engaging students in play, littleBits provided opportunities for students to discover computational technology insights, practices, and perspectives. Also the projects under the Micro:bit Educational Foundation [21] aim to bridge the gap between abstract data concepts and tangible experiences at the elementary and secondary school levels using a small programmable and embeddable computer-designed device.

Although sensor integration in education is beneficial, there is a lack of research in identifying the requirements for sensors that can be easily integrated into teaching. For sensor integration into school curricula, it becomes vital to analyze teachers' and students' needs and to define criteria for curricula-compatible sensors that could facilitate educational activities in suitable manner, for instance, being easy to handle, safe, simple in data presentations, etc. For this, we also have to classify sensors into relevant curricula-compatible classes that could be integrated with school subjects and we have to identify the requirements for such sensor classes under different conditions, i.e., indoor and outdoor. Moreover, analysis of existing sensors is also needed to determine whether they are school curricula-compatible or not and which adjustments are required to achieve the compatibility.

\section{Research Design and Methods}

The research study was carried out in two steps, R1 and R2, to investigate the stated research question (see Figure 1). In the first step, we identified the role that sensors might play in education, e.g., how sensor technology can be used for developing digital and data skills. We first identified the relevant curricula-compatible sensor classes that could be integrated into teaching as part of school subjects through interviews and a focus group meeting with teachers as participants. Then, the criteria for the attributes of the identified curricula-compatible sensors classes were defined based on teachers' feedback. In general, certain attributes must be 
considered when we choose a sensor, e.g., accuracy, environmental conditions, calibration, cost, and repeatability, as discussed in [9]. The second step R2, determines whether the existing sensors already used in educational activities satisfy the criteria for curricula-compatible sensors, and if not, what adjustments are needed to achieve this compatibility. Finally, requirements models for curricula-compatible sensors are developed under specific conditions, i.e., indoor and outdoor.

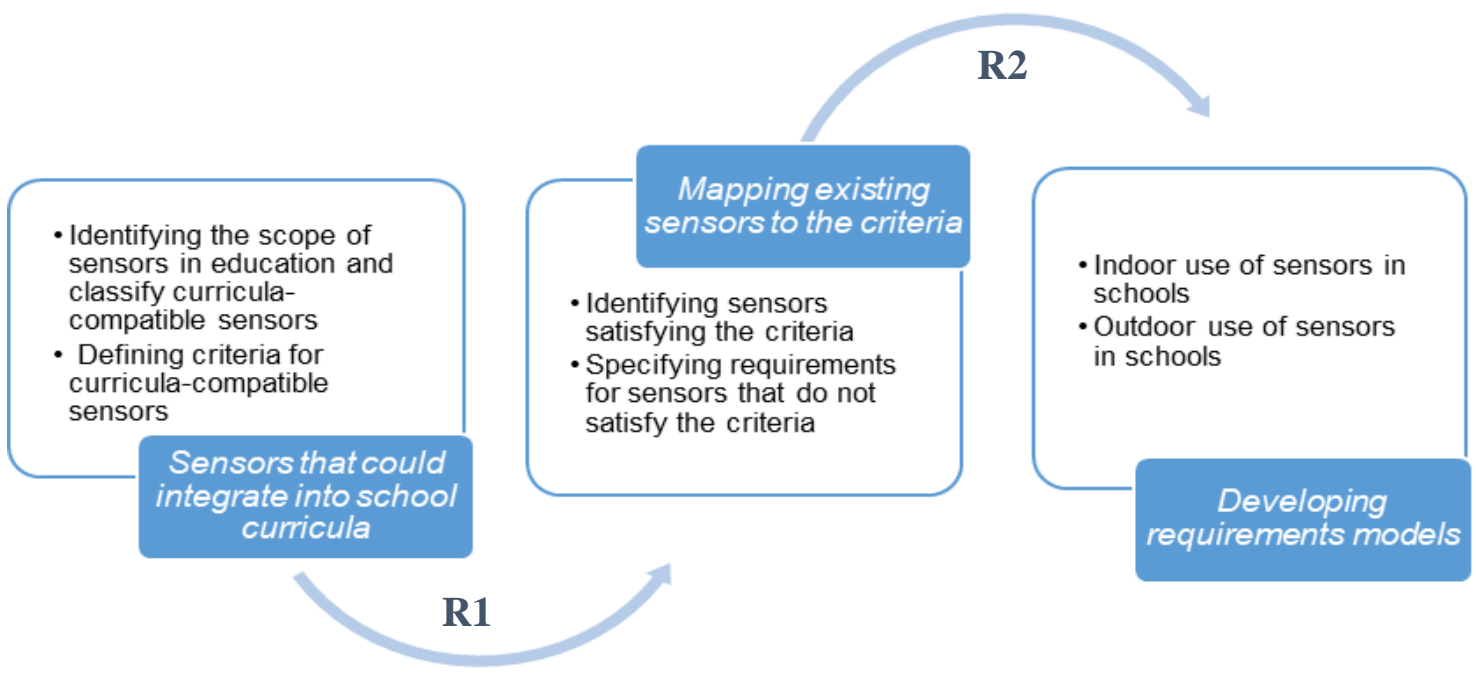

Figure 1. Research design to answer the research question "How can sensors facilitate the understanding of data and working with real world data, and how sensors can be designed for use in education?

\subsection{R1 - Criteria and Classes for Curricula-Compatible Sensors}

For revealing ways of integrating sensor technology into schools, we used one-on-one interviews and a focus group meeting methods. We approached 30 schoolteachers of grades 5-9 (students aged 11-15 years) and recorded their perspectives on the use of sensors in schools. We investigated how often they used sensors in their teaching, what made a sensor compatible for educational activities, and what were the requirements for such sensors. We also investigated why sensor use is essential in the educational domain and how students can take benefit from sensors as part of everyday learning activities. The focus group meeting and all the interviews were conducted in Danish and recorded with the teachers' consent. Participant details are given in Table 1, and procedure details are given below for each method.

Table 1. Participants' details

\begin{tabular}{|c|c|c|c|c|c|l|}
\hline Test setup & Participants & Schools & Grades & Subjects & Location & Duration \\
\hline $\begin{array}{c}\text { One-on-one } \\
\text { interview }\end{array}$ & 5 & 5 & $6-9$ & Math, science & $\begin{array}{c}\text { Aarhus, Aalborg, } \\
\text { Horsens }\end{array}$ & $1-1.5 \mathrm{hrs}$. \\
\hline Focus group & 25 & 12 & $6-9$ & Math, science & Aarhus & $1 \mathrm{hr}$. \\
\hline
\end{tabular}

One-on-one interview procedure: Interviews are considered a core method for eliciting requirements [22]. We conducted interviews to get the perspective of teachers in using sensors as part of school subjects, to identify problems in using existing sensors, and to determine how teachers can promote the frequent use of sensors for data-collection activities as part of teaching assignments. During the one-on-one interviews, questions were asked about how often teachers worked with sensors, what were the associated opportunities in using sensors as part of school activities, which types of sensor they used, and in which subjects they used sensors to collect real data. We asked about the various challenges they faced in using existing sensors and what attributes they thought were essential for curricula-compatible sensors. 
Focus group procedure: Focus groups help gather in-depth details about a group's actions, thoughts, and feelings, as participants can interact and converse with others while discussing a topic [23]. In our case, the focus group was a mix of teachers of different subjects (math, science, geography) and grades (5-9). The main aim was to investigate the use of sensors as an educational aid in schools, the curricula-compatible sensor classes that could facilitate secondary education in school subjects, and what specific attributes could make these sensors curriculacompatible. The meetings started with a short (10-minute) presentation about the possibilities of using sensors in everyday teaching in schools rather than just in laboratory assignments. Afterwards, the teachers participated in open discussions and discussed different aspects of sensors to be used in schools, sensor classes, and their attributes required for use in education. They also provided their responses in a posttest questionnaire (see Appendix A) about how often they used sensors in their teaching plans, which parameters they normally measured using sensors, and in which subjects they used sensors.

\subsection{R2 - Proposing Requirement Models for Curricula-Compatible Sensors}

In the second step R2, we tested curricula-compatible sensor classes as well as their attributes as classified by teachers in science subjects with students aged 11-15 years. The aim was to identify further requirements important for sensors' use in educational setting, i.e., under specific conditions indoor and outdoor. To achieve the aim we used the following method.

We ran pilot tests both indoors with 24 students and outdoors with 30 students to identify the requirements and challenges in using sensors as a tool to collect, analyze, and interpret data. Table 2 shows the participants' details. The pilot tests were conducted in Danish and recorded with the students' consent.

Table 2. Participants' details in pilot tests with students

\begin{tabular}{|c|c|c|c|c|c|c|}
\hline Sr. No. & Test Setup & Participants & Grade & Subject & Location & Duration \\
\hline Pilot test 1 & Indoor & 16 & 9 & Science & Aarhus & 6 hrs. \\
\hline Pilot test 2 & Outdoor & 16 & 9 & Science & Aarhus & $3 \mathrm{hrs}$. \\
\hline Pilot test 3 & Indoor & 8 & 7 & Science & Aarhus & $3 \mathrm{hrs}$. \\
\hline Pilot test 4 & Outdoor & 14 & 8 & Science & Aarhus & $3 \mathrm{hrs}$. \\
\hline
\end{tabular}

Indoor pilot test procedure: Indoor pilot tests were conducted at two different schools in 7th and 9th grade science lessons. For the indoor pilot tests, the teachers designed activities as part of science subjects in which students used temperature, pressure, and light sensors. The students collected data inside the classroom, such as light intensity under different conditions, e.g., with open and covered windows. They also recorded room light intensity and studied what light intensity levels were optimal for the classroom reading environment. Environmental sensors were also used to analyze how the classroom environment changed under different conditions, e.g., humidity, temperature, etc. Using temperature sensors, the students recorded classroom temperature at different locations and found the minimum and maximum temperature values. We used the observation method to identify difficulties when students worked with these sensors. Afterward, the students also answered a posttest questionnaire (see Appendix B) with both openended and closed questions regarding working with sensors.

Outdoor pilot test procedure: The science teachers for 8th and 9th grade students designed outdoor tests. The students went outside the classroom and collected data using temperature, light, pollution, and $\mathrm{pH}$ sensors. They also used an application for recording noise levels. Students recorded the temperature of stream water, checked its quality, and measured the light intensity under the shade and in direct sunlight. The students were very engaged, performed tasks in teams, and answered a questionnaire prepared by the teachers. Afterward, they also 
answered a posttest questionnaire (see Appendix B) with questions about the use of sensors and the data collected.

\section{Results}

In this section, we discuss the results obtained at research steps R1 and R2. Overall, the teachers agreed that learning could be strengthened using sensor technology that enables students to directly relate the subject to their surroundings. In general, teachers use sensor technology with secondary school students in physics, chemistry, and biology, particularly in technical experiments.

The teachers acknowledged that sensor technology and data collection activities are beneficial for elementary and secondary school students. However, they pointed also to challenges in the frequent use of existing sensors to facilitate educational activities, for instance, lack of resources, the extra time required to design activities, and unawareness of the possibilities that sensor technology can offer in teaching practices. There are also some barriers present due to the cost and maintenance of the sensors. Further in this section we discuss the following issues that yielded the main results of the study:

- The role of sensor technology in schools,

- Curricula-compatible sensor classes,

- Criteria for curricula-compatible sensors,

- Mapping to existing sensors,

- Requirements for curricula-compatible sensors under specific conditions.

The role of sensor technology in schools: Using one-on-one interviews and the focus group meeting in research step R1, we identified teachers' perspectives about integrating sensors as an educational aid to collect and analyze data as part of their subjects. Figure 2 represents how often teachers used sensors in their teaching plans. Most teachers used sensors to perform specific science experiments, but they also liked the idea of using sensors as educational instruments as part of everyday teaching to provide a hands-on experience to students and to relate the subjects to real data.

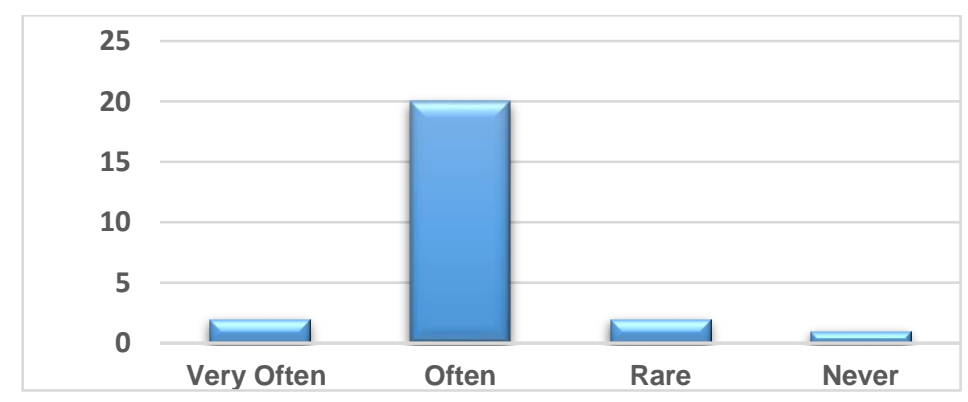

Figure 2. Teachers' frequency of use of sensors in teaching activities (the number on the Y-axis represents the number of teachers who responded to the focus group posttest questionnaire $N=25$ )

Figure 3 (see on the next page) represents various opportunities associated with sensor integration into schools according to the teachers' feedback during interviews and the focus group meeting.

According to the teachers, using the opportunities shown in Figure 3, students will learn more from the experience and retain more information about a topic, as sensors provide a hands-on experience in this regard and make the subject more interesting; also, students will participate more in discussions and understand better how data is generated, analyzed, and interpreted.

Curricula-compatible sensor classes: Table 3 shows the list of sensor classes with specific measuring properties that could easily be integrated into schools to collect data and give benefit to student learning in different subjects, e.g., measuring temperature, quality of light, etc. These 
classes were identified by teachers during one-on-interviews and focus group open discussion session.

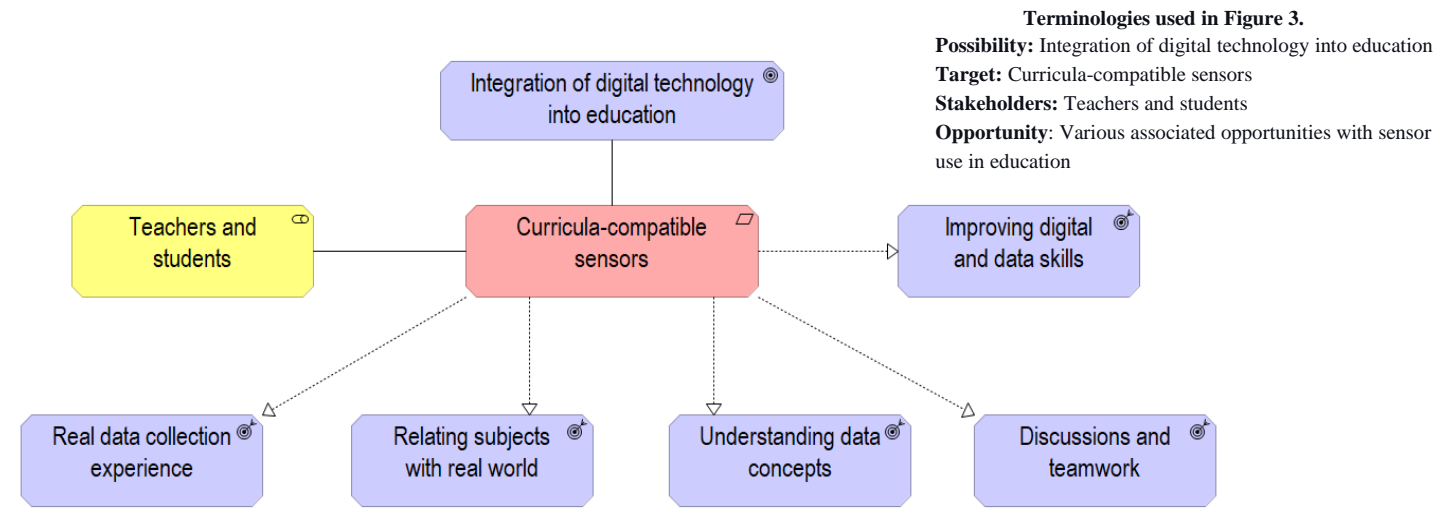

Figure 3. Associated opportunities with sensor use in education

Table 3. Curricula-compatible sensor classes identified from the one-on-interviews and focus group meeting $(N=30)$

\begin{tabular}{|l|l|}
\hline Educational sensor classes & Measuring properties \\
\hline Temperature sensors & Temperature, warmness, heat current, etc. \\
\hline Sound sensors & Sound, changes in sound pressure level, etc. \\
\hline Light sensors & Intensity of light, wavelength, polarity, etc. \\
\hline Pressure sensors & Pressure, force, etc. \\
\hline Environmental sensors & Humidity, air quality, CO level, etc. \\
\hline
\end{tabular}

Each class is briefly discussed below:

- Temperature sensors: A temperature sensor [24] is used to measure the amount of energy in the form of heat and cold produced by an object and a system. Temperature sensors make it possible to detect any physical change to that energy and give the output as an analog or digital signal. According to teachers, these sensors can attract students' attention in both science and math, e.g., they can measure the indoor temperature at different time intervals and can arrange data in tables and present the results.

- Sound sensor: A sound sensor is used to detect the intensity of sound. It converts the acoustic wave into an electrical signal output. Students can, e.g., use these sensors to monitor sound levels and note the noise level in the classroom.

- Light sensor: A light sensor is a photoelectric passive sensor that changes light energy into an electrical signal output. Light sensors can measure the ambient light, e.g., surrounding light, room light, and reflected light. Students can use the sensors to measure light intensity, quality of light, etc. to understand these abstract concepts using real data.

- Pressure sensor: Pressure is an external force exerted on a surface in unidirectional areas. In schools, generally, students can measure the pressure of a liquid, air, and other gases as part of a physics lesson, and pressure sensors can help students to understand the concept of pressure in a real setting.

- Environmental sensor: These sensors can be used, e.g., in classrooms to measure indoor climate, such as air quality, humidity, CO level, etc.

Figure 4 presents the list of educational sensor classes and their properties that could facilitate school subjects by relating real data to their respective subjects. The properties are also identified from the response of 30 teachers during one-on-interviews and focus group meeting. 


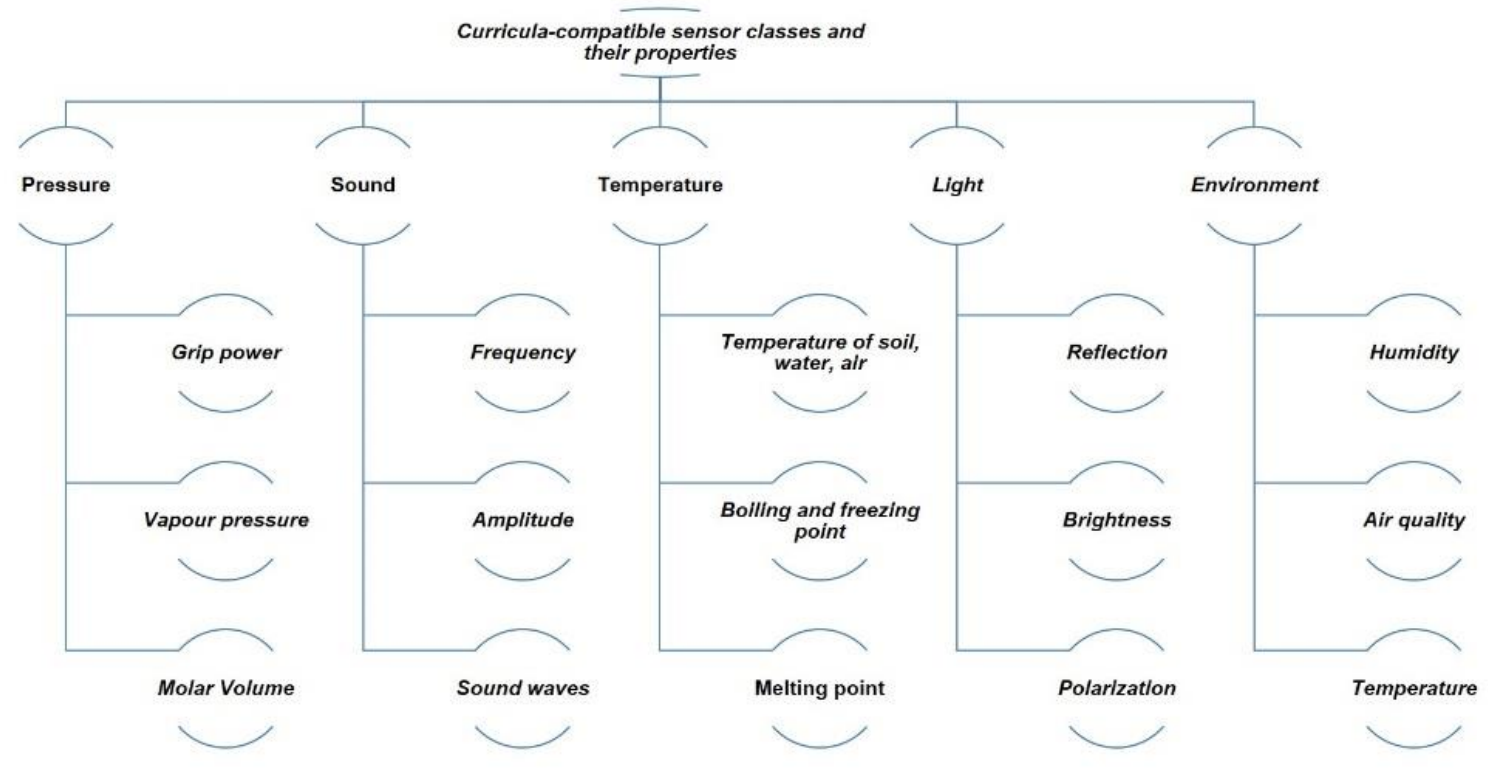

Figure 4. Curricula-compatible sensor classes and their properties $(N=30)$

General criteria for curricula-compatible sensors: While different sensor classes have their specific properties, in this research we were interested in criteria, which could be applied to sensors in general. We define curricula-compatible sensors as those sensors that can help with educational activities in the classroom. The key parameters that concern the generic characteristics of the curricula-compatible sensors, e.g., accuracy and safety, and so on, are referred to as curricula-compatible sensor attributes, and the corresponding values are referred to as attribute values. The attribute criteria for curricula-compatible sensors refer to the set of specific rules that attribute and their corresponding attribute values must satisfy. Curriculacompatible requirements are defined as the attributes that sensors must possess in order to be considered curricula-compatible sensors.

To define general criteria for curricula-compatible sensors, various attributes were selected from the literature [9], [25], [26] and verified with teachers during one-on-one interviews and the focus group meeting. A list of various attributes was presented to teachers during one-on-one interviews and focus group meetings. Teachers chose attributes that they believed were important in an educational setting in open discussion during focus group meeting. Figure 5 represents the identified attributes required for curricula-compatible sensors according to teachers' response. All of the listed attributes in Figure 5 are important in an educational setting, but some are more significant than others. For instance, safety and ease of handling are the main attributes that a sensor must have in order to be used in an educational activity. Furthermore, data representation software for curricula-compatible sensors is required for data to be easy to understand. The other attributes also have significance when working with students, such as response time, accuracy, range scale, and maintenance, as students can easily divert their attention if, e.g., the response time is too long. 


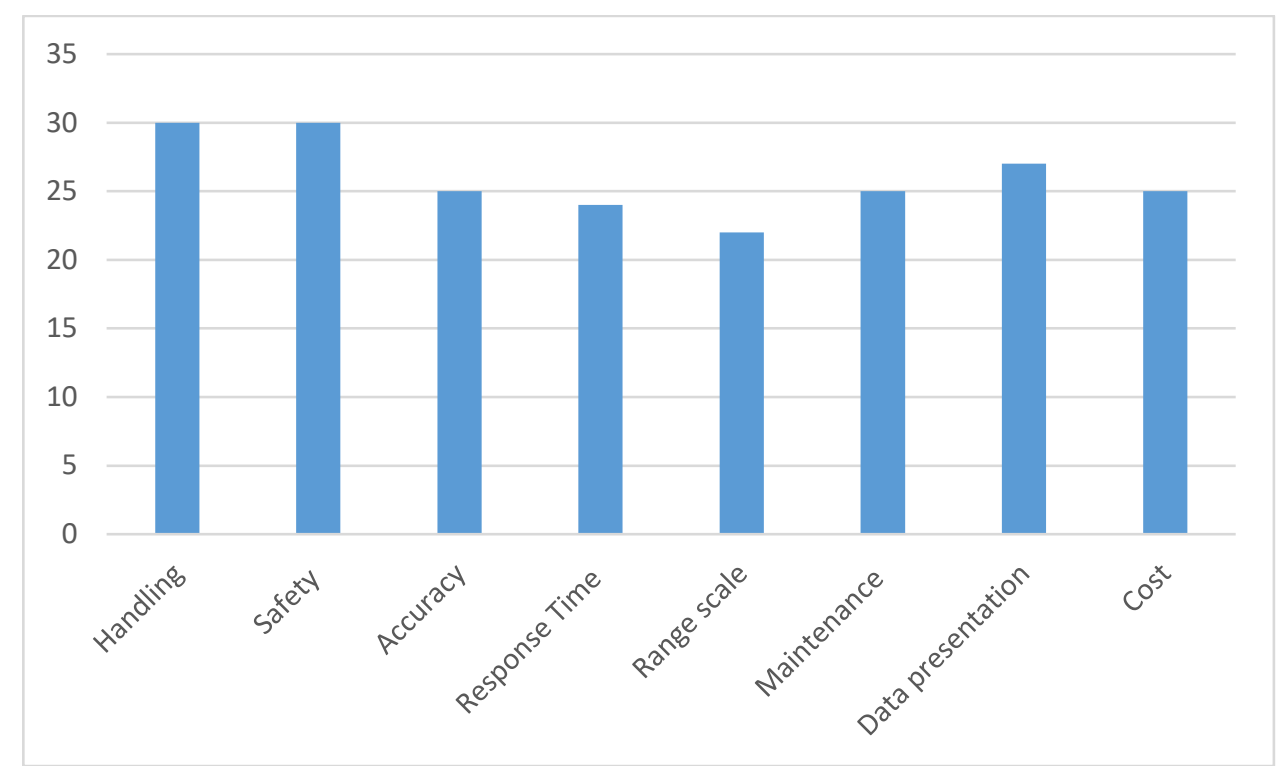

Figure 5. Teachers' perspective on sensor attributes where the $Y$-axis represents number of teachers participated in the focus group and one-on-interviews $(N=30)$

Figure 6 represents the obtained set of sensor attributes and the corresponding criteria for sensor attributes value, in general for being used in educational activities, based on teachers' feedback during one-on-one interviews and the focus group meeting.

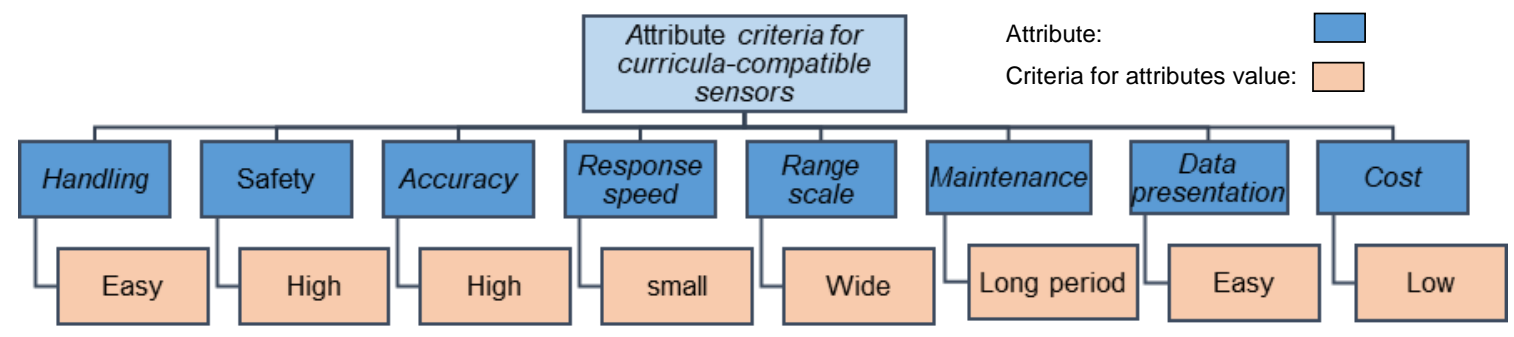

Figure 6. Curricula-compatible attributes and criteria for corresponding attributes value

The identified attributes are briefly described below:

- Handling: To integrate sensors into schools, students and teachers must use them without difficulties. According to the teachers' feedback, students will pay more attention to datacollecting activities rather than investigating how to use the sensor if the design of a sensor is simple.

- Safety: The teachers recognized safety as an important attribute for curricula-compatible sensors. For students aged 11-15 years, sensors must fulfill the criteria for curriculacompatible sensors. For instance, in case of damage or accident, sensors' safety attribute must be high. Hence, the material, design, and shape of a sensor must be harmless under any conditions.

- Accuracy: Accuracy is an important characteristic in sensors and is calculated in terms of the error in measurement and defined as the difference between the measured value and the true value of a measured parameter [27]. For a sensor to be curricula-compatible, it must measure value to an extent that allows students to relate real values of a parameter to their collected values. Teachers suggested that, if the measured value is close to the actual value, it would help to retain students' attention; otherwise, students may lose interest.

- Response speed: According to teachers, for data-collection activities in schools, response time should be minimal for a curricula-compatible sensor. 
- Range scale: The range scale is the difference between the maximum and minimum values of the sensed parameter. For a sensor to be used in education, it should have a wide operating range and good accuracy over the range.

- Maintenance: For a sensor to be used in education, it must require minimal maintenance over a long period of time.

- Data presentation: Software for data presentation and analysis must be simple and easy to use by both students and teachers.

- Low cost: The sensors must be affordable so that the sensors can easily be used in educational activities as part of everyday teaching.

Mapping curricula-compatible sensor criteria to existing sensors: Under this theme, existing sensors used in schools were identified and listed during the focus group meeting. We tested these sensors to determine whether they fulfilled the criteria of curricula-compatible sensors under different conditions, i.e., indoor and outdoor. During the indoor ( 24 students) and outdoor (30 students) pilot tests with students of 7th, 8th, and 9th grades, the students tested the sensors given in Table 4. The tasks were designed to test the curricula-compatible sensor attributes classified by teachers. Most of the existing sensors are expensive and sensitive when satisfying the other criteria such as handling, simple data presentation etc., and, therefore, do not fit well to be used as a tool for school activities. The safety and maintenance attributes of sensors are not represented in Table 4 because most existing sensors meet the criteria corresponding to them.

Table 4. Matching attribute criteria for curricula-compatible sensors with sensors (sensor classes) already available in the market

\begin{tabular}{|c|c|c|c|c|c|c|c|}
\hline $\begin{array}{l}\text { Existing } \\
\text { Sensors }\end{array}$ & 昜 & $\frac{3}{\overparen{E}}$ & 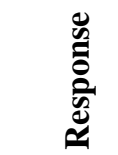 & $\overrightarrow{8}$ & 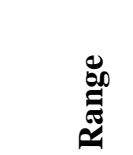 & 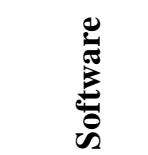 & $\begin{array}{l}\text { Suggested adjustment } \\
\text { for curricula- } \\
\text { compatible sensors }\end{array}$ \\
\hline $\begin{array}{l}\text { Temperature } \\
\text { sensors }\end{array}$ & Easy & High & Quick & Low & Wide & Simple & Fulfilled criteria \\
\hline Barometers & Difficult & High & Quick & High & Wide & Difficult & $\begin{array}{l}\text { Cost should be adjusted } \\
\text { and handling and data } \\
\text { presentation should be } \\
\text { simplified }\end{array}$ \\
\hline Light sensors & Easy & Moderate & Quick & High & Wide & Difficult & $\begin{array}{l}\text { Data presentation } \\
\text { should be simpler, and } \\
\text { cost should be adjusted }\end{array}$ \\
\hline $\begin{array}{l}\text { Indoor } \\
\text { environment } \\
\text { sensors }\end{array}$ & Easy & Moderate & Slow & High & Limited & Simple & $\begin{array}{l}\text { Response time and cost } \\
\text { should be adjusted }\end{array}$ \\
\hline $\begin{array}{l}\text { Sound } \\
\text { sensors }\end{array}$ & Easy & Low & Quick & High & Wide & Difficult & Cost should be adjusted \\
\hline Microphones & Easy & High & Quick & Low & Wide & Simple & Fulfilled criteria \\
\hline $\begin{array}{l}\text { Outdoor } \\
\text { environment } \\
\text { sensors }\end{array}$ & Easy & Moderate & Slow & High & Limited & Simple & Cost should be adjusted \\
\hline
\end{tabular}

Teachers used these existing sensors in laboratories with great caution, and the students did not get a chance to explore data-related activities in an independent environment. We discovered that the sensors' attributes were either directly or indirectly related with each other; for instance, if a sensor satisfied the criteria for handling, safety, and accuracy, then the criterion for cost was not fulfilled. For instance, the cost of sensors that are easy to handle, safe, and have high accuracy is much higher than of one with low accuracy and easy handling. However, the 
attribute values could be adjusted in existing sensors to transform them into curricula compatible sensors. For instance, the high cost of environmental sensors can be adjusted by providing only the features that are useful for school teaching instead of those for professional use, such as accuracy up to two decimal places.

Requirements for curricula-compatible sensors under specific conditions: The criteria defined above represent general attributes that curricula-compatible sensors must have; however, there are some specific requirements sensors must have to be considered as curricula-compatible sensors under specific conditions, such as indoor and outdoor. These requirements were identified during pilot tests with students and are discussed below.

Indoor: To identify the requirements for indoor use of sensors in schools, we used wireless temperature, light, and pressure sensors. We chose these sensors as they were frequently used by teachers. We observed students during the tests, for instance, how they handled the sensors, what difficulties they faced while working with the sensors, and their data-collection activities. The model shown in Figure 7 describes the requirements we identified during the two indoor pilot tests at different schools in the 7th and 9th grades.

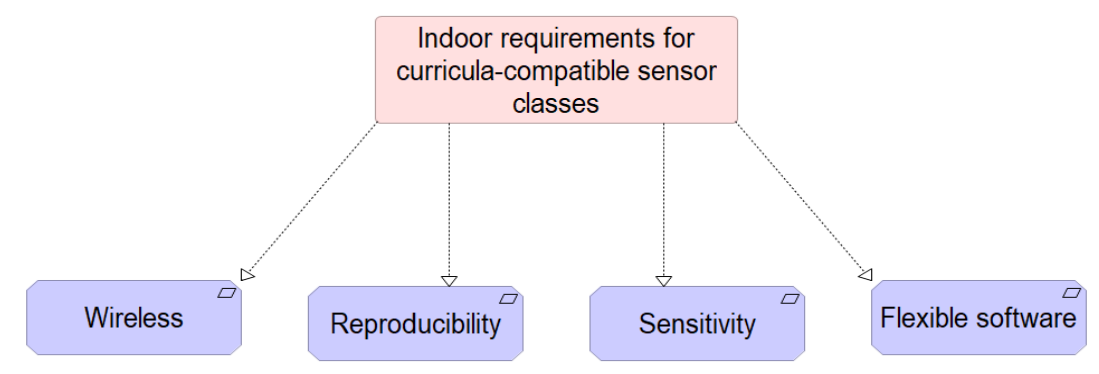

Figure 7. Requirements for indoor curricula-compatible sensors.

The students also answered a short online survey with questions focusing on various problems they faced. For instance, students faced difficulties in connecting the sensors to data-analytic software. For light sensors, the students recorded different data under the same circumstances. It was difficult for them to understand which data were correct and why they had different data recorded by the same sensor. Furthermore, teachers emphasized the need for a flexible data analysis software that collects data from various types of sensors and is simple to install.

Outdoor: One of the main requirements identified during the pilot tests with students (grades 8 and 9) concerned the environmental conditions while collecting data outside the building. The students used light sensors, pollution sensors, $\mathrm{pH}$ sensors, and a noise application for data collection. According to teachers' feedback during the focus group meeting and observation during outdoor tests, the students faced difficulties in collecting data due to environmental factors such as temperature, corrosion, pressure, etc. Environmental conditions were the main factors that affected the input and output stimuli of the sensors. Noise also affected the output signal of the sensors in some cases. Therefore, for sensors to be curricula-compatible, the performance and long-term stability of the sensors should be considered for extreme conditions so that these factors do not create adverse effects during the data-collection activity in schools. Figure 8 represents a requirement model for the outdoor use of sensors.

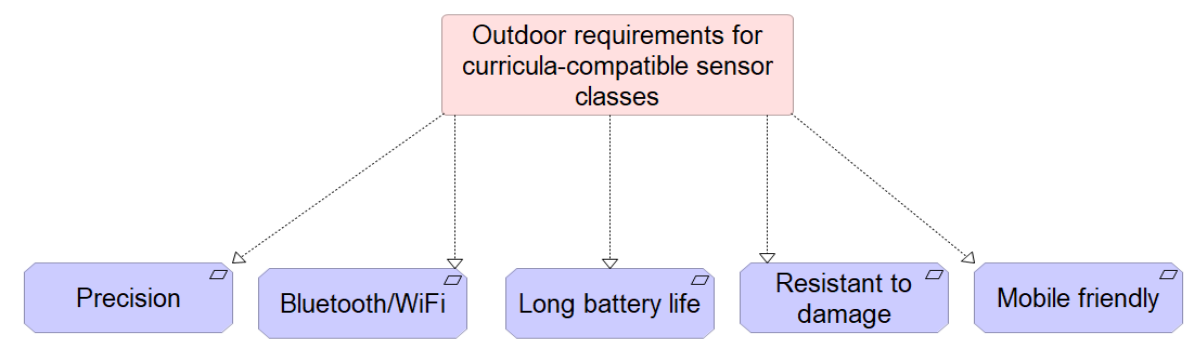

Figure 8. Requirements for outdoor curricula-compatible sensors. 
Teachers also suggested that if the sensors could connect and send the collected data to any device or application compatible with mobile phones, it would make the data collection process simpler.

\section{Discussion and Conclusions}

In this article, we studied how sensors can facilitate the understanding of data and working with real data as well as how curricula-compatible sensors should be designed. Sensors are useful for incorporating computing and technology into the curriculum for school students. Using sensors in the classroom helps to bring science lessons to life and builds confidence in using technology that is not typically found in the classroom. We learned that existing sensors have some barriers to their frequent use in schools; for instance, if safety and easy handling are of main priority, then the cost is high. Also, in some cases, the data presentation software was not compatible with the school computer system.

This study was limited to only some schools in Denmark. Nevertheless, it provides school administration and teachers with an overview of why and how to integrate sensors into school education. For instance, the criteria enable teachers to consider the various attributes essential for sensors before choosing a sensor for a subject. The results of the study also enable manufacturers to build sensors that meet the criteria defined in this study for educational purposes. For instance, high professional quality is not required for sensors to be used in education; rather, they must have certain attributes that should meet the criteria for curricula-compatible sensors. The analysis of related work, sensor specifications, the results of five teacher interviews, the focus group (25 teachers), and four pilot tests (two indoor tests with 24 and two outdoor tests with 30 students) led to the following conclusions:

1. The use of sensors in schools can facilitate the understanding and handling of real data in the following ways:

- It allows students to work with real data collection,

- It helps to relate teaching subjects to the real world,

- It supports the understanding of the concept of data,

- It promotes decision-making and teamwork,

- It improves the digital skills of students.

2. Five relevant curricula-compatible sensor classes are identified, i.e., temperature sensors, sound sensors, light sensors, pressure sensors, and environmental sensors, that could facilitate educational domains in developing digital and data skills among school students.

3. Curricula-compatible sensors should have the following attributes: easy handling, high safety, high accuracy, moderate response speed, a wide range scale, easy maintenance over a longer period, easy data presentation, and low cost.

Further analysis of different classes of available outdoor and indoor sensors made it possible to define specific requirements for curricula-compatible sensors that are amalgamated in two requirements models. The results of the sensor analysis also showed whether adjustments are needed to existing sensors to apply them in school curricula. We found that, at present, only temperature sensors and microphones fulfill the criteria for curricula-compatible sensors.

The findings reflected in this article are useful for understanding current possibilities and identifying which future developments are needed regarding the integration of use of sensors into school curricula.

Based on the findings of this study, our future research will focus on how to combine sensor data collected during educational activities with open educational datasets to facilitate engaging teaching assignments in schools. 


\section{Acknowledgements}

We acknowledge stakeholders' contribution to this research work, including the Community Drive project and Aalborg University for providing funding, Danish public schools, teachers, and students. We also acknowledge the valuable comments of the anonymous reviewers of this article.

\section{References}

[1] E. Worthen, "Sensory-based interventions in the general education classroom: A critical appraisal of the topic," Journal of Occupational Therapy, Schools, \& Early Intervention, vol. 3, no. 1, pp. 76-94, 2010. Available: https://doi.org/10.1080/19411241003684217

[2] T. Bell, D. Marghitu, and L. Lambert, "Computer science unplugged, robotics, and outreach activities," in Proceedings of the 43rd ACM Technical Symposium on Computer Science Education, p. 657, 2012. Available: https://doi.org/10.1145/2157136.2157332

[3] M. Saddiqa et al, "Bringing open data into Danish schools and its potential impact on school pupils," in Proceedings of the 15th International Symposium on Open Collaboration, pp. 1-9, 2019. Available: https://doi.org/10.1145/3306446.3340821

[4] M. Saddiqa et al., "Enterprise Architecture Oriented Requirements Engineering for the Design of a School Friendly Open Data Web Interface," Complex Systems Informatics and Modeling Quarterly, vol. 21, pp. 1-20, 2019. Available: https://doi.org/10.7250/csimq.2019-21.01

[5] M. Andrée, "Ways of using 'Everyday life' in the science classroom," in Research and the Quality of Science Education, pp. 107-116, 2005. Available: https://doi.org/10.1007/1-4020-3673-6_9

[6] K. Mayoh and S. Knutton, "Using out-of-school experience in science lessons: reality or rhetoric?" International Journal of Science Education, vol. 19, no. 7, pp. 849-867, 1997. Available: https://doi.org/10.1080/0950069970190708

[7] J. Atenas, L. Havemann and E. Priego, "Open data as open educational resources: Towards transversal skills and global citizenship," Open Praxis, vol. 7, no. 4, pp.377-389, 2015. Available: https://doi.org/10.5944/openpraxis.7.4.233

[8] National Academies of Sciences, Engineering, and Medicine, Science and Engineering for Grades 6-12: Investigation and Design at the Center. 2019.

[9] J. Fraden, Handbook of Modern Sensors. Springer, 2013.

[10] A. S. Bos et al, "Educational technology and its contributions in students' focus and attention regarding augmented reality environments and the use of sensors," Journal of Educational Computing Research, vol. 57, no. 7, pp. 1832-1848, 2019. Available: https://doi.org/10.1177/0735633119854033

[11] M. C. Diamond, "Response of the Brain to Enrichment," International Journal of Interdisciplinary Education, vol. 1, no. 1, 2012.

[12] P. Howard-Jones, J. Taylor and L. Sutton, "The effect of play on the creativity of young children during subsequent activity," Early Child Development and Care, vol. 172, no 4, pp. 323-328, 2002. Available: https://doi.org/10.1080/03004430212722

[13] A. E. McFarlane, "Developing an Understanding of the Meaning of Line Graphs in Primary Science Investigations, Using Portable Computers and Data Logging Software," Journal of Computers in Mathematics and Science Teaching, vol. 14, no. 4, pp. 461-480, 1995.

[14] S. Gallagher, "Collecting and manipulating weather data: using technology tools in a first-grade classroom," Computers in the Schools, vol. 16, no. 3-4, pp. 167-175, 2001. Available: https://doi.org/10.1300/J025v16n03_05

[15] C. T. Nicolaou et al., "Enhancing fourth graders' ability to interpret graphical representations through the use of microcomputer-based labs implemented within an inquiry-based activity sequence," Journal of Computers in Mathematics and Science Teaching, vol. 26, no. 1, pp. 75-99, 2007. 
[16] A. A. Zucker et al., "Learning science in grades 3-8 using probe ware and computers: Findings from the TEEMSS II project," Journal of Science Education and Technology, vol. 17, no. 1, pp. 42-48, 2008. Available: https://doi.org/10.1007/s10956-007-9086-y

[17] M. Perrin, "Inquiry-based pre-engineering activities for K-4 students," Journal of STEM Education: Innovations and Research, vol. 5, no. 3, 2004.

[18] S. T. Chen et al., "ASPECT sinking and floating: An interactive playable simulation for teaching buoyancy concepts," in Proceedings of the First ACM SIGCHI Annual Symposium on Computer-Human Interaction in Play, pp. 327-330, 2014. Available: https://doi.org/10.1145/2658537.2662978

[19] K. Grufberg and M. Jonsson, "Sciensations: Making sense of science by designing with sensors," in Proceedings of the 11th International Conference on Interaction Design and Children, pp. 116-124, 2012. Available: https://doi.org/10.1145/2307096.2307110

[20] V. Lin and O. Shaer, "Beyond the lab: Using technology toys to engage South African youth in computational thinking," in Proceedings of the 2016 CHI Conference Extended Abstracts on Human Factors in Computing Systems, pp. 655-661, 2016. Available: https://doi.org/10.1145/2851581.2851589

[21] J. Austin et al., "The BBC micro: bit: from the UK to the world," ACM, vol. 63, no. 3, pp. 62-69, 2020. Available: https://doi.org/10.1145/3368856

[22] A. Bryman, Social Research Methods. 2016.

[23] A. Parker and J. Tritter, "Focus group method and methodology: current practice and recent debate," International Journal of Research \& Method in Education, vol. 29, no. 1, pp. 23-37, 2006. Available: https://doi.org/10.1080/01406720500537304

[24] R. Usamentiaga et al., "Temperature measurement using the wedge method: Comparison and application to emissivity estimation and compensation," IEEE Transactions on Instrumentation and Measurement, vol. 60, no. 5, pp. 1768-1778, 2010. Available: https://doi.org/10.1109/TIM.2010.2089894

[25] J. E. Brignell and N. M. White, Intelligent Sensor Systems: 2nd ed. 1996.

[26] W. Heerens, “Application of capacitance techniques in sensor design,” Journal of Physics E: Scientific Instruments, vol. 19, no. 11, p. 897, 1986. Available: https://doi.org/10.1088/0022-3735/19/11/002

[27] K. Tseng, C. Huang and F. Lian, "Analysis of sensor characteristics and error eliminations of a wheeled mobile robot with range sensors," in 2008 IEEE International Conference on Robotics and Biomimetic, pp. 228-233, 2009. Available: https://doi.org/10.1109/robio.2009.4913008 


\section{Appendix A}

Questionnaire on the use of sensors in schools to collect data (Translated from Danish)

\section{Personal Information}

School name:

Gender:

Age:

Experience:

What grade levels do you teach?

1. What pedagogical approaches do you use in your teaching and how much?

- Traditional direct instruction

- Teaching with experiments

- Collaborative learning

2. Have you ever used sensors or technology (e.g., mobile applications, trackers) in your teaching activities?

- Yes

- No

3. To what extent do you use sensors in teaching?

- More often

- Often

- Rare

- Never

4. What parameters do you measure with sensors in your teaching activities most often?

- Temperature

- Pressure

- Light

- Sound

- Position

- Motion

- Environmental parameters e.g. CO, moisture etc.

- Other

5. Are the currently used sensors and associated software easy to understand and use by both you and your students? Briefly explain your answer.

6. How sensors use can be made easy in educational activities, i.e. easily used by students and teachers (simple graphs, sensors design or shape)?

7. Are some school subjects better suited to the integration of sensors than others? If so, which ones? 


\section{Appendix B}

\section{Pilot Test Survey 2020 (Translated from Danish)}

School name:

Grade:

1. Which of the following methods do you like best when collecting data?

- With notebook

- With sensors

- With mobile application

2. Do you like working with sensors and technology?

- Yes

- No

3. What is difficult when working with sensors?

- Set up the sensor

- Software

- Understand graphs

- Understand data

4. Is the light sensor easy to use?

- Yes

- No

5. What difficulties do you encounter when working with sensors, such as the software, graphs, or the sensor itself?

- I do not know how to use the sensor without the help of the teacher

- Connecting the sensors to software is difficult

- You must be very careful when using sensors

- Other (please specify)

6. Do you need help all the time when working with sensors?

- Yes

- No

7. Did any problems occur while working with the temperature sensor, such as the sensor not responding or collecting the correct data?

- Yes

- No.

- If so, please mention

8. What parameters do you like to measure using sensors?

- Noise / noise

- Temperature / heat / cold

- Physical activities

- Air quality

- Other things. 